\title{
An Ontology Development For HACCP Knowledge Description and Sharing in Food Cold Chain
}

\author{
Mu Xiangwei, Zhang Lin \\ Transportation Management College \\ Dalian Maritime University \\ Dalian, China,116026 \\ muxiangwei@dlmu.edu.cn
}

\begin{abstract}
In order to achieve an accurate expression and effective sharing about the hazards monitoring and managing knowledge between the upstream and downstream of the cold chain, the paper establish a HACCP ontology model of the cold chain on the basis of analyzing and summarizing general business processes of the cold chain and HACCP management system.In the experiment, we implement the HACCP ontology of the cold chain according to the background knowledge of the application, and achieve automatic identification of the concept-instance by applying the SWRL inference rules and HermiT inference engine, and share business knowledge among the different steps in the cold chain. The results show that the model can be applied to model the HACCP management knowledge to improve the management efficiency of the cold chain hazard monitoring.However, due to the complexity of the cold chain monitoring and managing, the model still requires combining with the knowledge in the field of specific domain in the processes of practical application to further improve.
\end{abstract}

Keywords- cold chain logistics; ontology; HACCP; knowledge description; knowledge reasoning;

\section{INTRODUCTION}

With food safety incidents increasing significant all over the world, the food safety surveillance has become a hot topic with high profile. In order to ensure that the food is safe and controllable in the whole process, In 1997, the CodexAlimentarius Commission announced "International Recommended Operating Standard The Profile Of Food Hygiene”, the standard stipulates critical control measure at each stage form primary production to final consumption, and use the Hazard Analysis and Critical Control Points(HACCP) method to make the food safe.

HACCP has become internationally recognized and accepted as the food safety assurance system, it is widely used in food, pharmaceuticals and other products in the cold chain business links. However, the construction of cold chain logistics needs a huge investment, many food producing and processing enterprises trend to use the third-party cold chain logistics service[1,2], how to ensure the integrity of the multi-party cold chain logistics safety monitoring is becoming an important research field in recent years.Aninformation architecture was analyzed and established for third-party refrigerated warehousing companies to improve operational efficiency and reliability of third-party refrigerated storage[3].A framework based on Delphi-AHP-TOPSIS method was proposed to assess the effects and defects during the cold chain operating procedure, and it point out that the most difficult is to collect key information in the process of practical application [4].According to relevant studies, the key issue to make sure that the entire cold chain process safety is to ensure that the critical information of monitoring and managing during every processes in the cold chain is flowing and sharing.

This paper attempts to use the Semantic Web Ontology language, OWL2DL establish cold chain HACCP ontology, CC-HACCP Ontology, in order to compensate for the deficiencies in the ability of rule description and ratiocinate semantic, the model also use the production rules based on SWRL and logic-based inference engine, realize the formal representation and inference of the knowledge, such as service features, experience, and rules. The model can be applied to the knowledge semantic descript and share about the cold chain hazards monitoring and managing to help managers improve the cold chain HACCP management system, and the correct implementation of HACCP in all aspects of the cold chain.

\section{HACCP}

Hazard analysis and critical control points, referred to as HACCP.is made up of two parts of Hazard Analysis and Critical Control Point.The core idea of the HACCP management system is to analysis the raw materials, production processes and the impact of product safety human factors, to identify key processes in the machining process, to establish and perfect monitoring program and monitoring standard, and to take the standardize and corrective measures.HACCP's purpose is to eliminate the possible occurrence of food safety hazards in the production process, rather than by the post test to ensure the safety of food.It consists of seven basic elements [5]: the hazard analysis (HA), and to determine the potential hazards in each stage of the food production, including raw materials processing, manufacturing, storage products, sales and other processes; the determination of the critical 
control points (CCP), it can be a controlled point, step or method ,through controlling the food potential hazards can be prevented, eliminated to an acceptable level; the key limit value (CL), for each of the CCP need to determine a standard value, to ensure that each CCP limit value in the security value; establishing the CCP monitoring program; set up and take effective corrective measures; establish a verification procedures, including the confirmation of the plan, CCP verification and HACCP system verification, to verify the HACCP system and the relevant results have reached the expected effect; establish effective record keeping procedures, to provide the basis for improvement and subsequent control.

\section{COLD CHAIN HACCP ONTOLOGY}

In order to make HACCP key information to share better in the cold chain between the upstream and downstream, HACCP system is also the comprehensive of experience and knowledge of cold chain related decision makers in practice accumulation, in recent years, the rising ontology theory and correlation method has become one of effective means of supply chain knowledge retrieval and sharing, such as: Xiaohuan Wang, et al [6]studied on global collaborative decisionmaking knowledge of supply chain ,and established knowledge decision model based on ontology and rules. Wang Gong, et al [7] built a multi-agent system based on the ontology, which can model and reason the related strategic knowledge of the cooperative negotiation.BENDRISS Sabri et al [8] establish a general information model for the supply chain product traceability using the Semantic Web ontology model .

The knowledge and the key information can be in the smooth flow of the upstream and downstream and sharing for the cold chain monitor hazards management is particularly important in the Cold chain management of HACCP process,we can reference successful application experience of ontology theory and method in the field of supply chain , build the ontology model of describing cold chain of HACCP management system of knowledge.)

In this paper, the Description Logic SROIQ(D)[9] language is used to describe the HACCP knowledge of cold chain.Respect to the grammatical format of Semantic Web ontology language OWL 2 DL[10], the SROIQ(D) can not only makes the knowledge description more concise and owl 2 is based on the SROIQ(D), convenient into the format using the computer to process the owl 2 DL syntax more easily.

Cold Chain HACCP Ontology is defined as four tuple: $C C-H A C C P=\{C, A, P, I\}$

Where $\mathrm{C}$ represents a collection of concepts, A represents a collection of data types, $\mathrm{P}$ is a collection of objects, and I represents an instance of the collection.

\section{A. the Basic Concepts of CC-HACCP}

The basic concept set $\mathrm{C}$ of CC-HACCP, $C_{C C}$ ${ }_{H A C C P}$, Contains the hazard analysis and critical control points concept of HACCP, cold chain concept of the subject of CC_Role, cold chain goods concept CC_Cargo and cold chain business process concept $C \bar{C} \_$Step, describing logic grammar is described as follows:
$C_{C C-H A C C P}=\left\{H_{A C C P}, C C_{-}\right.$Role,$C C_{-}$Cargo, CC_Step, $C C_{-}$Proof $\}$ $\boldsymbol{H A C C P} \subseteq \boldsymbol{C C P} \sqcup \boldsymbol{H A} \sqcup \boldsymbol{C M} \sqcup \boldsymbol{C L} \sqcup \boldsymbol{C A} \sqcup \mathrm{VE}$

CC_Role $\subseteq$ Raw_Material_Supplier $\sqcup$ Producer $\sqcup$ Logistics_Service_Provider $\sqcup$ Wholesaler $\sqcup$ Retailer

CC_Cargo $\subseteq$ Frozen_Cargo $\sqcup$ Refrigerated_Cargo

Hazard analysis and critical control point concept HACCP. According to the related knowledge of hazard analysis and critical control point system we can be obtained the HACCP concept is made of the critical control point CCP , hazard analysis $H A$ and control measures $C M$, key limit value concept $C l$, corrective measures $C A$, verification $\mathrm{VE}$, and records $R E$.

Cold chain subject concept CC_Role. According to the analysis of the cold chain of business process. In this paper, cold chain is divided for the following roles: Raw_Material_Supplier, Logistics_Service_Provider,Wholesaler and Retailer.

The concept of CC_Cargo can be divided into two types of Frozen_Cargo and Refrigerated_Cargo by storage temperature range. Where the frozen type of goods is required to store, transport, wholesale and distribute in the following $-18^{\circ} \mathrm{C}$. While cold type of goods is required in the following $7^{\circ} \mathrm{C}$.

Business process concept CC_Step. Step is the abstract in the concrete steps of the cold chain business process, Step are responsible for the implementation and operation by CC_Role.

Monitoring object information carrier concept CC Proof, monitoring object information carrier, is the important evidence used to detect whether meet the key limit value ,the fact may include: the goods state with appearance of the cargo and the packing seal , official proof materials such as proof of inspection and quarantine or non-epidemic areas,cargo loading and unloading records and goods out of warehouse ledger and other business operation records, warehousing and transportation environment temperature records and the relevant records $(R E)$ produced by HACCP system.

\section{B. Basic Properties of CC-HACCP}

The relationship of the cold chain HACCP ontology is mainly divided into two kinds of object attributes and data attributes. Object properties mainly describe the relationship between the concepts, the scope and range are concepts, part of CC-HACCP's objects properties are show in Tab.1.

TABLE I. OBJECTS PROPERTIES OF CC-HACCP

\begin{tabular}{|c|c|c|}
\hline Objects Properties & Domain & Range \\
\hline next_step & CC_Step & CC_Step \\
\hline is_charged_by & CC_Step & CC_Role \\
\hline handle_cago & CC_Step & CC_Cargo \\
\hline cause_by & HA & CL \\
\hline has_CA & CL & CA \\
\hline
\end{tabular}

data propertiesdescribe the relationship between the concept and the data type, the scope is concept, the range is related data types, including: integer, string, float, Boolean, symbol, XML scheme datatype and so on, part of CC-HACCP's data properties are show in Tab.2. 
TABLE II. DATA PROPERTIES OF CC-HACCP

\begin{tabular}{|c|c|c|}
\hline DataProperties & Domain & Range \\
\hline package_type & CC_Cargo & string \\
\hline quality_guarantee_period & CC_Cargo & float \\
\hline has_tempure_limits & CL_Tempure & integer \\
\hline has_date_limits & CL_Time & date \\
\hline
\end{tabular}

\section{CC-HACCP Expansion}

In order to further improve the CC - HACCP, HACCP management system should converse the principles, regulations and business rules of knowledge into cold chain concept of ontology model and semantic constraints, attribute features elements

1) Concept Expansion

According to the HACCP principle 3 and cold-chain actual knowledge of the business, the key limit (CL) usually adopts the temperature, time, humidity, the examination index and sensory parameters,such as appearance and organization form. The CL concept increase the following concepts:

\section{$C L \subseteq$}

CL_Cargo $\cup$ CL_Documents $\cup$ CL_Temperature $\cup$

CL_Time U CL_Index;

\section{CL_Index $\equiv$ Biological_Index $\cup$ Chemical_Index $\mathrm{U}$} Physical_Index;

\section{2) PropertyConstraints}

According to the specified in the HACCP management system,for each key control pointmust provide critical limit, monitoring measures need tobein a planned way to measure or observe the key control points of key limit value, and the monitoring process documentation.According to the above knowledge can produce constraints is as follows:

$C C P \equiv C C \_S t e p \cap \exists h a s_{-} C M . C M$;

$C M \subseteq H A C C P \cap \exists$ monitor_CL. $C L \cap \exists h a S_{-} R E . R E$

3) PropertyFeatures

Such as object property "next_step" describes the logical sequencebetween the cold chain business step, so it istransitive, thus add the transitiveFeatures of the object attribute next_step.

$$
\text { next_step }{ }^{+} \sqsubseteq \text { next_step; }
$$

\section{Inference Rules}

CC-HACCP has certain reasoning ability by semantic meaning, but limited to the description logic, it can not describe the Association Rules (If. Then), event flow and query inference.In this paper, SWRL,Semantic Web Rule Language[118], is used to add the semantic description into CC-HACCP.

The general form ofSWRL rules is" santecedent $\rightarrow$ consequent", where both antecedent and consequent are conjunctions of atoms written "atom1,atom2,...atom3”. Variables are indicated using the standard convention of prefixing them with a question mark "?x". Due to space limitations, this article only list the following three rules.

\section{- Step Generate Proof Rule}

If cold chain in a step implemented a monitoring measures $\mathrm{cm}$ and $\mathrm{cm}$ have records(re), and re can be used to test a critical limit $(c l)$, thenreshould be consider as a instance of CC_Proof, and is generated in the step. In fact, the deduction that the $r e$ is a instance of $C C$ _proofinference is not obtained from the direct inference of the rule, but because the rules using the step'sobject attributes step_generate_proof, which range is CC_proof. So we can infer that the re is an instance of the proof of concept CC_proof. The rules are described as follows:

has_CM(?step, ?cm),has_RE(?cm, ?re),CL_proved_by(?cl,?re)

$$
\rightarrow \text { step_generate_proof(?step, ?re) }
$$

\section{- Step Submit Proof Rule}

If cold chain in a steps step1 implemented a monitoring measures $\mathrm{cm}$, and $\mathrm{cm}$ monitoring key limit value of $\mathrm{Cl}$ and proven track record by the monitoring object information carrier proof, andstep2 and step1 are not the same steps, and in the cold chain process, step1 is after step2, then step2 need to submit proof which generated in step2toits subsequent business steps.Because of the next_stepistransitive,next_step(step2,step1) is trueeven if thestep1 is notdirectly following the step2. The rules are described as follows:

has_CM(?step1, ?cm), monitor_CL(?cm, ?cl), CL_proved_by(?cl, ?proof), proof_from_step(?proof, ?step2), DifferentFrom (?step1, ?step2), next_step(?step2, ?step1) $\rightarrow$ step_submit_proof(?step2, ?proof)

\section{- Step Deliver Proof Rule}

Based on the Rule of Step Submit Proof Rule expression, if there is step3 which is different amongstep1 and step2, and step3 is intermediate step between Step2 and step1, then step3 need todeliver the proof. The rules are described as follows:

$$
\begin{gathered}
\text { has_CM(?step1, ?cm), } \\
\text { monitor_CL(?cm, ?cl),CL_proved_by(?cl, ?proof), } \\
\text { proof_from_step(?proof, ?step2), DifferentFrom } \\
\text { (?step1, ?step2), DifferentFrom (?step1, ?step3), } \\
\text { DifferentFrom (?step2, ?step3), CC_Step(?step3), } \\
\text { next_step(?step2, ?step3), next_step(?step3, ?step1) } \\
\rightarrow \text {-step_deliver_proof(?step3, ?proof) }
\end{gathered}
$$

\section{EXPERIMENT}

The case's background is the cold chain of eating oyster products. In this case, cold chain process include: Live oyster fishing, live oysters transportation, raw material receiving, production and processing. Live oysters supplier did not establish the HACCP system, The third cold chain logistics service provider and the producer preliminary establishesHACCP management system, but the information and knowledge is not shared, such as, the logistics service providerdoes not know whatinformationis required by the producer.

\section{A. Experiment 1: CC-HACCP Ontology Building}

Combinedrelevant standards, CC-HACCPbased on OWL 2 DL language was built by using the ontology tool protege5, the class structureis shown in Fig.1. 


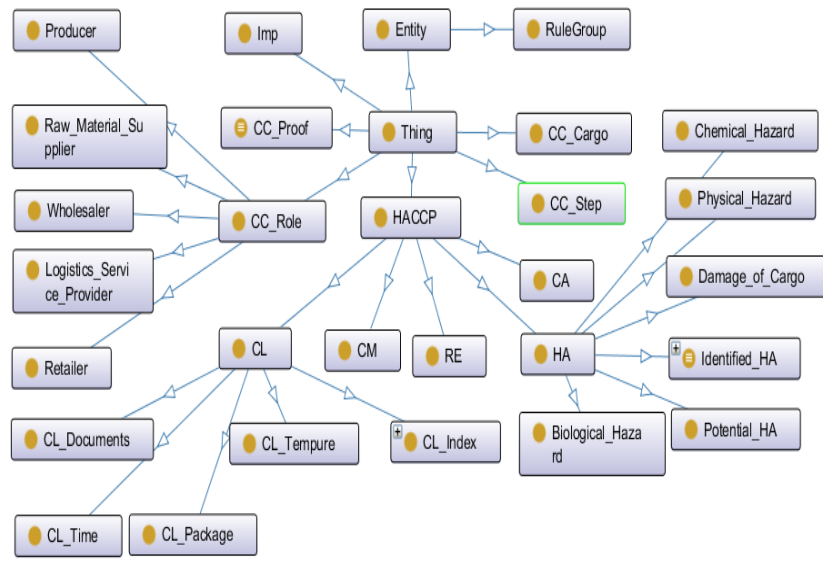

Figure 1. CC-HACCP Class Structure.

\section{B. Experiment 2: Inference Basd on CC-HACCP}

The inference engine HermiT (version 1.3.8) reasoning, which accord to the definition of CC-HACCP, constraints and characteristics of properties, was used to realize the concept of consistent test and instanceautomatic identification. Such as an instance of the concept of CC_Step, "step_of_live_oyster_transportation" was identified as the critical control points (CCP)and the "Risk_Step" automatically. The reasoning result is shown in area 1 of Fig.2.

At the same time, inference machine according to the inference rules presented in the previous paper, deduce the new object property assertionsbetween instances of various concepts, which is unknown before the reasoning,such as: "step_of_live_oyster_transportation" which is an instance of CC_Stepget some object properties after the reasoning, the result is shown in the area 2 of Fig.2.

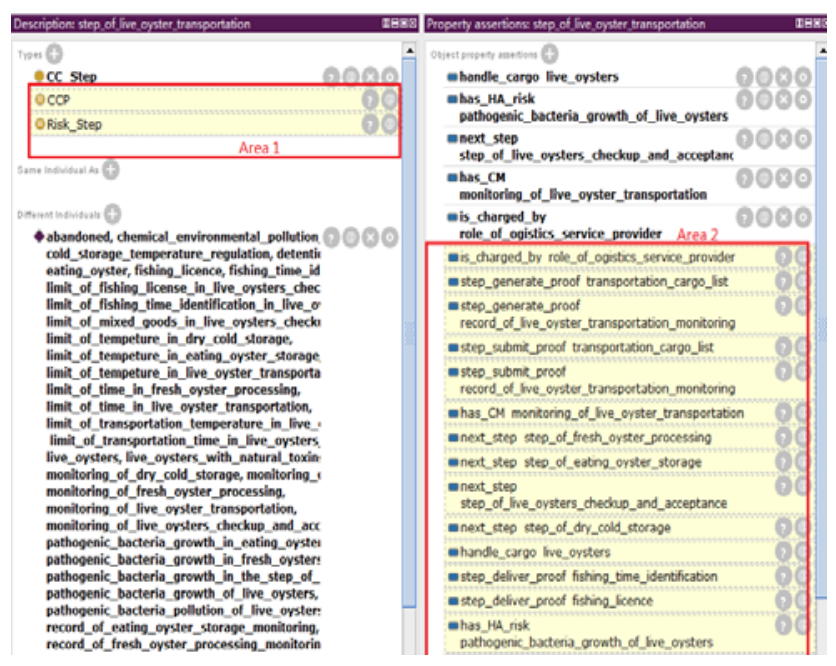

Figure 2. Example of Inference Result.

The reasoning results show that the CC_Step“step_of_live_oyster_transportation"should submit

theCC_Proof'transportation_cargo_list"and"record_of_li ve_oyster_transportation_monitoring",which was generated by itself.This step was also required to deliver the "fishing_time_identification" and "fishing_license" to the next step, which were generated by the the previous step of "step_of_live_oysters_Fishing".

Based on the inference engine, the reason of inference result can be explained. There were two ways to explain why CC_Step of "step_of_live_oyster_transportation" is also an instance of $C C P$, as shown in Fig.3.

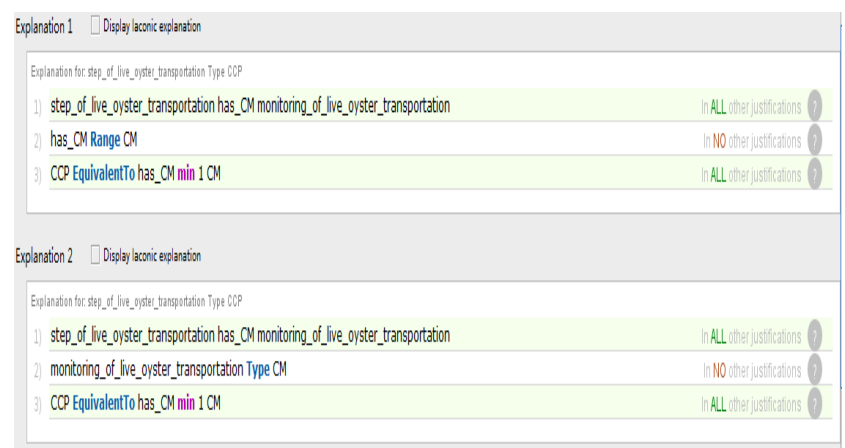

Figure 3. Example of Inference Result Explanation.

\section{Experimental Summary}

Experimental results show that CC-HACCP ontology can be well used to build the smantic knowledge model in the actual business process of the cold chain,which will help the cold chain manager tooptimize the HACCP system in multiparty participation cold chain, and share the HACCP knowledge.

\section{ACKNOWLEDGMENT}

Project funded by China Postdoctoral Science Foundation(2014M551063), Scientific Research Fund of Liaoning Provincial Education Department(L2014203), Liaoning Social Science Planning Fund (L14BGL012) and the Fundamental Research Funds for the Central Universities(3132015050).

\section{REFERENCES}

[1] Z. Z. Liu, Study on cold chain of China's food industry[J]. Logistics Technology, 2006,8:67-68.

[2] Chen Na, Shi Lei. Analysis of Quality Game Behavior for ColdChain Logistics Outsourcing[C]//International Conference on Logistics Systems and Intelligent Management[s.n.], 2010:202-206.

[3] Zhang Qin, Zhang Keming, Song Bohui.The Information Construction of Third-Party Warehousing in the Cold Chain Logistics[C]//2nd International Conference on Logistics, Informatics and Service Science[s.n.], 2013: 409-414。

[4] Rohit Joshi, D.K. Banwet, Ravi Shankar. A Delphi-AHP-TOPSIS based benchmarking framework for performance improvement of a cold chain[J]. Expert Systems with Applications .2011,38:10170 10182.

[5] WorldHealth Organization. Application of Hazard Analysis and Critical Control Point (HACCP) methodology to $\begin{array}{ll}\text { pharmaceuticals[EB/OL]. } & \text { (2003)[2015]. }\end{array}$ http://www.who.int/medicines/areas/quality_safety/quality_assuran ce/ApplicationHACCPMethodologyPharmaceuticalsTRS908Anne x7.pdf?ua=1.

[6] Xiaohuan Wang, T.N. Wong, Zjhi-Ping Fan. Ontology-based supply chain decision support for steel manufacturers in China[J]. Expert Systems with Applications, 2013,40:7519-7533.

[7] Gong Wang, T.N. Wong, Xiaohuan Wang. An ontology based approach to organize multi-agent assisted supply chain negotiations[J]. Computers \& Industrial Engineering, 2013,65:2-15. 
[8] BENDRISS Sabri, BENABDELHAFID Abdellatif. Enabling product traceability through data modeling and semantic web service ontologies[C]//2013 International Conference on Advanced Logistics and Transport[s.n.], 2013:365 - 370.

[9] HorrocksI, KutzO, SattlerU. The even more irresistible SROIQ[J]. In KR, 2006:57-67.
[10] G. Antoniou,P. Groth, F. van Harmelen, R.Hoekstra. A Semantic Web Primer[M], 3rd ed.,China: Machine Press,2014:54-72.

[11] Ian Horrocks, Peter F. Patel-Schneider, Harold Boley, Said Tabet, Benjamin Grosof, Mike Dean. SWRL: A Semantic Web Rule Language Combining OWL and RuleML[EB/OL]. W3C Member Submission , (2014-4-21).http://www.w3.org/Submission/SWRL/. 